\title{
Observation of fast spontaneous emission decay in GalnAsP photonic crystal point defect nanocavity at room temperature
}

\author{
Toshihiko Baba, ${ }^{a)}$ Daisuke Sano, Kengo Nozaki, Kyoji Inoshita, and Yusuke Kuroki \\ Yokohama National University, Department of Electrical and Computer Engineering, 79-5 Tokiwadai, \\ Hodogayaku, Yokohama 240-8501, Japan \\ Fumio Koyama \\ Tokyo Institute of Technology, Precision and Intelligence Laboratory, 4259 Nagatsuta, Midoriku, Yokohama \\ 226-8503, Japan
}

(Received 29 March 2004; accepted 27 August 2004)

\begin{abstract}
The spontaneous emission decay in a photonic crystal slab nanocavity with a GaInAsP quantum well active region was measured at room temperature. Even far below lasing threshold, the decay was much faster than that for the as-grown wafer. A consideration including the enhanced spontaneous emission rate by the Purcell effect, intraband relaxation of carriers, nonradiative surface recombination, spatial hole burning, and carrier diffusion enabled us to explain different decay lifetime between on- and off-resonant conditions and between different size cavities. As a result, $>16$-fold shorter spontaneous emission lifetime was estimated, which strongly suggests the existence of a large Purcell effect. (C) 2004 American Institute of Physics. [DOI: 10.1063/1.1811379]
\end{abstract}

Purcell predicted that the spontaneous emission (SPE) rate is essentially enhanced in a high $Q$ microcavity. ${ }^{1-3}$ This so-called Purcell effect has been experimentally studied in semiconductor microcavities such as microdisks and multilayer stacks with an (AlGa)InAs quantum-dot (QD) active region at cryogenic temperature. ${ }^{4-6}$ The two level system of electrons in QDs and the suppression of nonradiative recombinations at cryogenic temperature simplified the evaluation of this effect, and a Purcell factor $F$ (degree of the enhancement) of 2-15 was estimated. However, a more common situation in microcavity studies is those with a quantum well $(\mathrm{QW})$ active region at room temperature. Previously, we reported the observation of Purcell effect in a GaInAsP compressively strained (CS) QW microdisk at room temperature. ${ }^{7}$ The InP based material is advantageous due to its small surface recombination velocity. ${ }^{8}$ In that study, the shortening of carrier lifetime was observed in the phaseresolved photoluminescence (PL) measurement. By considering the intraband relaxation in continuous electronic bands of QWs and the surface recombination at disk surface and sidewalls, $F$ was estimated to be 6.7. In this letter, we demonstrate a larger Purcell factor in a photonic crystal (PC) point defect nanocavity, which has an ultrasmall effective cavity volume (modal volume). ${ }^{9-11}$ The time-resolved measurement was carried out instead of the phase-resolved one to observe directly the SPE decay. Consequently, faster decay was observed particularly under on-resonant condition. One could consider that the result is similar to that for the QD active region at cryogenic temperature. However, this letter describes a more complex picture, which relates Purcell effect with spectral and spatial dynamics of carriers and explains observed phenomena more reasonably.

In the experiment, two different cavities were fabricated into a triangular lattice PC slab, i.e., H1 cavity of single missing airhole and $\mathrm{H} 7$ cavity of 127 missing airholes with three crossing line defects, as shown in Fig. 1. In H1, six innermost airholes are made smaller to improve the $Q$

\footnotetext{
${ }^{a)}$ Electronic mail: baba@ynu.ac.jp
}

factor. ${ }^{11}$ In $\mathrm{H} 7$, three line defects are used as photonic band gap mirrors at a narrow cutoff frequency range of line defects, which is effective for the limitation of resonant modes. ${ }^{12,13}$ Structural details and the fabrication process are the same as those in 13; the lattice constant is $0.44 \mu \mathrm{m}$, the airhole diameter $0.24 \mu \mathrm{m}$, the slab thickness $0.25 \mu \mathrm{m}$, and the active layer a GaInAsP CS QWs with a PL peak wavelength $\lambda=1.58 \mu \mathrm{m}$. The photopump measurement of fundamental lasing characteristics was carried out at room temperature with $\lambda=0.98 \mu \mathrm{m}$ pulsed laser light (75 ns pulse width, $10 \mathrm{kHz}$ repetition frequency, and 5-6 $\mu \mathrm{m}$ focused spot diameter). The lasing wavelength is around $1.6 \mu \mathrm{m}$, and

(a)
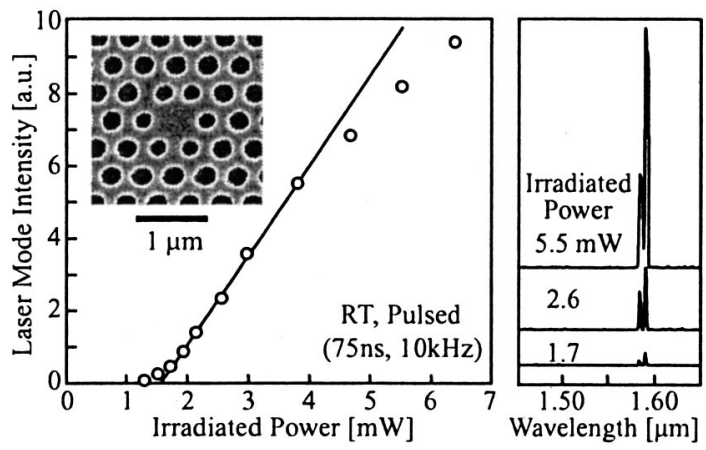

(b)
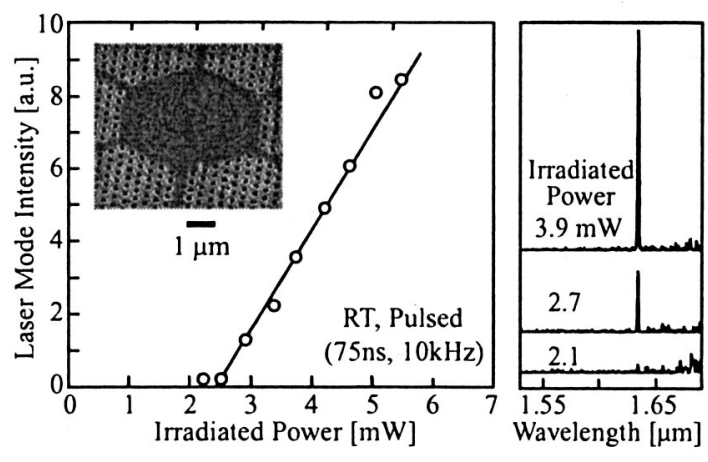

FIG. 1. Lasing characteristics of (a) $\mathrm{H} 1$ and (b) $\mathrm{H} 7$ cavities. Inset shows scanning electron microscope pictures of the same type devices. 
(a)

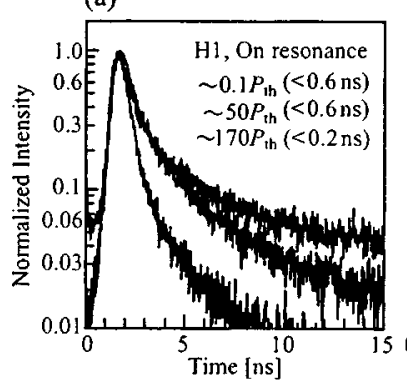

(b)

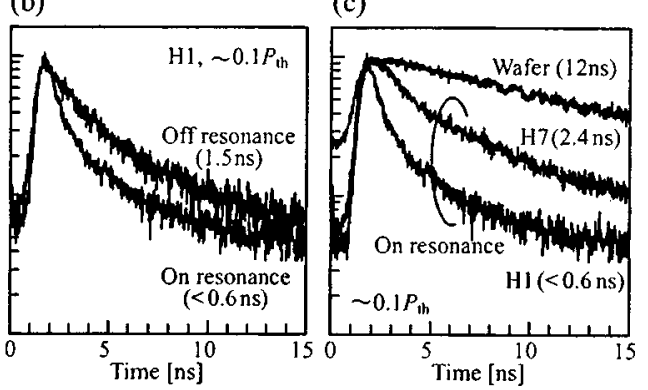

FIG. 2. Observed emission decay. (a), (b), and (c) compare different pump levels, on- and off-resonant conditions, and different samples, respectively. $P_{\text {th }}$ means the threshold power roughly estimated for $\mathrm{H} 1$ cavity by considering differences of the pump peak power and spot diameter between this measurement and the measurement for Fig. 1, and by comparing the pulse width and carrier lifetime (see Ref. 14). Here, the carrier lifetime is not the shortened lifetime by the Purcell effect but the lifetime simply determined by the wafer lifetime and the nonradiative lifetime (see Ref. 7). It is assumed to be $3 \mathrm{~ns}$ as a typical value. the threshold irradiated peak power $P_{\text {th }}$ are 1.5 and $2.5 \mathrm{~mW}$ for $\mathrm{H} 1$ and $\mathrm{H} 7$ cavities, respectively. The threshold for $\mathrm{H} 1$ is higher than those in previous papers, ${ }^{9-11}$ due to the wider pump spot diameter and the insufficient optimization of the cavity. Observed double peak spectrum and a finite difference time domain (FDTD) simulation indicate that the lasing was obtained by dipole modes. From expanded double peaks observed below threshold, $Q$ was roughly evaluated to be 1000. On the other hand, H7 exhibits the single mode lasing. However, three or more resonant modes were observed below threshold against the continuous wave pumping even with the three line defects.

In the measurement of the SPE decay at room temperature, yttrium-aluminum-garnet laser light at $\lambda=1.06 \mu \mathrm{m}$ ( $0.56 \mathrm{~ns}$ pulse width and $12.52 \mathrm{kHz}$ repetition frequency) was used for pumping. The focused spot diameter was expanded to $\sim 17 \mu \mathrm{m}$ to avoid the burn-in of the device surface. This diameter almost covers the whole area of a device. As a detector, a photomultiplier tube (Hamamatsu Photonics R5509-72) was used, which was mounted on a monochrometer with $1 \mathrm{~nm}$ resolution. The output signal was evaluated by photon counting, and the decay lifetime was obtained by deconvolution analysis against the pump pulse. The time resolution of the measurement is $\sim 1 \mathrm{~ns}$, and the fastest limit of evaluated lifetime is $0.2-0.6 \mathrm{~ns}$, which depends on the noise floor level. The detected power is moderately weakened so that the photon counting condition is maintained. Figure 2(a) shows the decay for H1 under on-resonant condition against various pump levels $P$. Here, $P \sim 0.1 P_{\text {th }}$ is lower than the population inversion level. At far above and below the threshold, the decay lifetime reached the fastest limit $0.2-0.6$ ns. Figure 2(b) shows the difference between on- and off-resonant conditions at $P \sim 0.1 P_{\text {th }}$, where offresonance means $\sim 15 \mathrm{~nm}$ shorter wavelength than the resonant mode. The off-resonant condition exhibited a longer lifetime of 1.5 ns. Figure 2(c) compares results for H1, H7, and the as-grown wafer under on-resonant condition. The lifetime for the latter two are 2.4 and $12 \mathrm{~ns}$, respectively.

First of all, the short lifetime of $<0.6 \mathrm{~ns}$ even at $P$ $\sim 0.1 P_{\text {th }}$ strongly suggests the existence of the Purcell effect. This expectation is supported by the difference between onand off-resonant conditions. However, the story is not the same as that for the QD active region at cryogenic temperature. Figure 3 schematically illustrates the difference between the stimulated emission (STE) in a normal laser and the enhanced SPE by the Purcell effect in a microcavity laser. Above threshold, the carrier density is cramped (therefore, $\tau_{\mathrm{sp}}$ is fixed) in a normal laser, and the fast decay by the STE occurs regardless of $\tau_{\mathrm{sp}}$. On the other hand, even with the Purcell effect, the modified SPE lifetime $\tau_{\mathrm{sp}}^{\prime}$ is given by the sum of the SPE rate for all optical modes due to the fast Downloaded 05 Nov 2004 to 133.34.23.211. Redistribution subject intraband relaxation of carriers with a typical lifetime of 0.3 ps. $^{7}$ This means that no differences should be observed between on- and off-resonant conditions; fast decay by the Purcell effect should occur even under off-resonant condition.

Another candidate phenomenon that explains the observation is the spatial hole burning of carriers. Because of the large pump spot, detected light includes emissions from inside and outside of the cavity. However, the resonant emission must be coming from the cavity. If the resonant mode suffers Purcell effect, excited carriers in the cavity will disappear quickly and form a spatial hole. Figure 4 shows twodimensional carrier distributions after pumping, which are simulated by the FDTD-like analysis of laser rate equations including the cavity mode profile, the Purcell effect, the surface recombination, and the carrier diffusion. ${ }^{15}$ Here, standard material parameters ${ }^{16}$ including a typical carrier diffusion constant $D$ of $2 \mathrm{~cm}^{2} / \mathrm{s}, Q=1000$, and a low pump level below the population inversion level are assumed. In addition, normal SPE and 60-fold faster SPE are assumed for carriers spatially overlapping with the laser mode in Figs. 4(a) and 4(b), respectively. For the normal SPE, the carrier density takes maximum in the cavity where the surface recombination is relatively low. For the faster SPE, a clear spatial hole appears in the cavity due to the fast carrier decay. Once the spatial hole is formed, the decay lifetime is limited by the carrier diffusion from outside of the cavity. On the other hand, what we observe under off-resonant condition is thought to be not the off-resonant emission from the cavity but the emission from the wide area outside of the cavity. The decay lifetime on this condition is determined by $\tau_{\mathrm{sp}}$ of the material (nearly equal to the wafer lifetime $\tau_{\mathrm{wf}}$, surface recombination lifetime $\tau_{\mathrm{sf}}$ at sidewalls and surfaces of the PC slab, and the carrier diffusion into the cavity. However, the
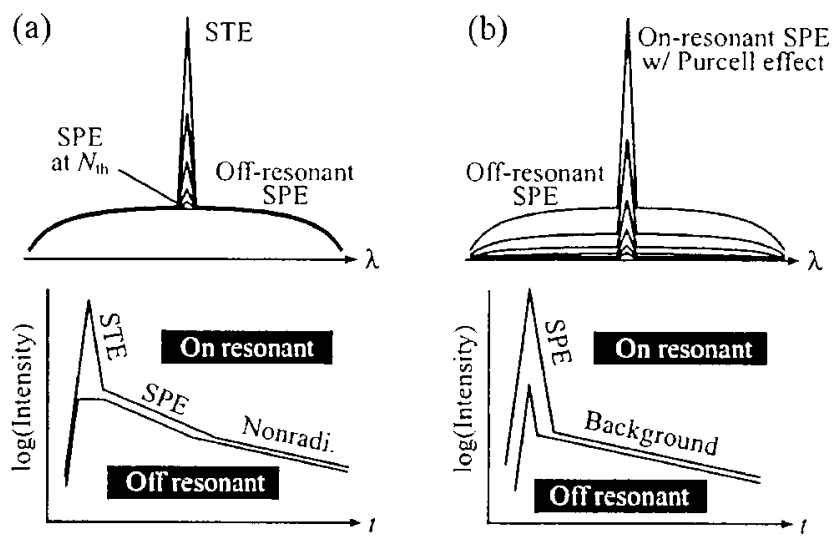

FIG. 3. Schematics of emission decay by (a) STE in a normal laser and (b)

SPE with the Purcell effect in a microcavity.
to AIP license or copyright, see http://apl.aip.org/apl/copyright.jsp 


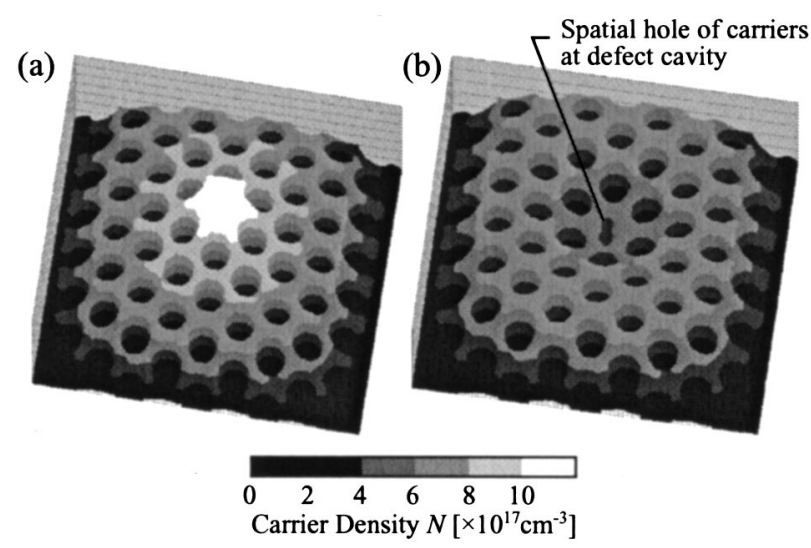

FIG. 4. Simulated carrier density distribution in H1 cavity maintaining a dipole mode with (a) normal SPE and (b) 60-fold faster SPE.

dominant factor is the surface recombination. In our previous work, we experimentally evaluated the surface recombination velocity $\nu_{s}$ for a PC based on the same material system to be $1.2 \times 10^{4} \mathrm{~cm} / \mathrm{s}^{8}$. From this value and the volume to surface ratio of the PC slab, $\tau_{\text {sf }}$ is calculated to be $1.5 \mathrm{~ns}$. It agrees well with the measured value for the off-resonant condition, and supports the earlier discussion. Let us check $\tau_{\text {sf }}$ in the cavity. Accounting cavity areas to be from the defect to half of the innermost airholes, $\tau_{\mathrm{sf}}$ is calculated to be 2.7 and $6.6 \mathrm{~ns}$ for $\mathrm{H} 1$ and $\mathrm{H} 7$, respectively. Assuming $\tau_{\mathrm{sp}}=\tau_{\mathrm{wf}}$, the modified SPE lifetime $\tau_{\mathrm{sp}}^{\prime}$ in these cavities are estimated to be $<0.8$ and $3.7 \mathrm{~ns}$, respectively, which mean $>16-$ and 3 -fold shortening of $\tau_{\mathrm{sp}}$.

According to the FDTD simulation, dipole modes in the H1 cavity have a very small modal volume $V$ of $0.085 \mu \mathrm{m}^{3} \sim 0.4(\lambda / n)^{3}$. With this value and $Q=1000$, the Purcell factor $F \sim 160$ is estimated from the simple formula $F=\left(p \Gamma_{r} \lambda^{3} / 4 \pi^{2} n^{3} V\right) Q{ }^{7}$ where $p$ is the polarization anisotropy factor ( $=1.4$ for the CS QW, i.e., $70 \%$ in-plane polarization) and $\Gamma_{r}$ is the relative confinement factor $(=1.7)$. It is not easy to confirm this value in the experiment, since $F$ is related with $\tau_{\mathrm{sp}}^{\prime}$ as $\tau_{\mathrm{sp}}^{\prime}=\tau_{\mathrm{sp}} / \Sigma F C$, where $C$ is the SPE factor and $\Sigma$ is the summation with respect to all modes in the SPE spectrum. For H7, the multiple resonances complicate the evaluation of $F$ and $C$ for each mode. Even for H1, $C$ was difficult to evaluate from the normal curve fitting method due to the weak light intensity below threshold. Regarding this cavity, Vučković et al. calculated $C \sim 0.4 \times 2$ for two dipole modes and in-plane polarized emission with a typical spectral width of $80 \mathrm{~nm} .{ }^{17}$ Even by considering the polarization anisotropy, we can expect $C \sim 0.3 \times 2$. From this value and the shortening of $\tau_{\mathrm{sp}}, F>27$ is estimated. This low value compared with the earlier estimation may be partly explained by the fastest limit of the measurement. Another possible explanation is the influence of the homogeneous broadening $\Delta E_{h}$ of electronic transition. The typical value of $\Delta E_{h}$ for GaInAsP at room temperature is $4.3 \mathrm{meV}$, and so $\Delta E_{h} / E$ $=0.0057 .^{7}$ Since it is wider than the cavity resonance, it can be a dominant factor, which changes the formula as $F$ $=\left(p \Gamma_{r} \lambda^{3} / 4 \pi^{2} n^{3} V\right) \cdot\left(E / \Delta E_{h}\right)$. According to the formula, $F$ is calculated to be $\sim 28$, which is much closer to the experimental value. This result suggests that, for the Purcell effect at room temperature, the small $V$ is more important than the high $Q$.

In conclusion, $>16$-fold faster SPE, which was limited by the response of the detector and/or the homogeneous broadening of electronic transition, was experimentally confirmed in the GaInAsP PC nanocavity. This means that a subnanosecond response is possible even without STE, the population inversion, and intentional nonradiative recombinations.

The authors would like to thank Dr. M. Notomi, NTT, for valuable discussion. This work was supported by the IT Program and the 21st COE Program of MEXT. The GrantIn-Aid of JSPS, and the CREST Project of JST.

${ }^{1}$ E. M. Purcell, Phys. Rev. 69, 681 (1946).

${ }^{2}$ E. Yablonovitch, J. Opt. Soc. Am. B 10, 283 (1993).

${ }^{3}$ T. Baba, IEEE J. Sel. Top. Quantum Electron. 3, 808 (1997).

${ }^{4}$ J. M. Gerard and B. Gayral, J. Lightwave Technol. 17, 2089 (1999).

${ }^{5}$ W. Fang, J. Y. Xu, A. Yamilov, H. Cao, Y. Ma, S. T. Ho, and G. S. Solomon, Opt. Lett. 74, 948 (2002).

${ }^{6}$ L. A. Graham, D. L. Huffaker, and D. G. Deppe, Appl. Phys. Lett. 74, 2408 (1999).

${ }^{7}$ T. Baba and D. Sano, IEEE J. Sel. Top. Quantum Electron. 9, 1340 (2003).

${ }^{8}$ H. Ichikawa, K. Inoshita, and T. Baba, Appl. Phys. Lett. 78, 2119 (2001).

${ }^{9}$ M. Loncar, T. Yoshie, A. Scherer, P. Gogna, and Y. Qiu, Appl. Phys. Lett. 81, 2680 (2002).

${ }^{10}$ H.-Y. Ryu, S.-H. Kim, H.-G. Park, J.-K. Hwang, and Y.-H. Lee, Appl. Phys. Lett. 80, 3883 (2002).

${ }^{11}$ H.-Y. Ryu, M. Notomi, E. Kuramoti, and T. Segawa, Appl. Phys. Lett. 84, 1067 (2004).

${ }^{12}$ K. Inoshita and T. Baba, Electron. Lett. 39, 844 (2003).

${ }^{13} \mathrm{~K}$. Inoshita and T. Baba, IEEE J. Sel. Top. Quantum Electron. 9, 1347 (2003).

${ }^{14}$ Y. Suematsu and S. Akiba, Trans. Inst. Electr. Eng. Jpn., Sect. E 59, 1 (1976).

${ }^{15}$ K. Nozaki and T. Baba, Int. Sympo. Photonic and Electromag. Crystal Structures, 2004, p. Th-P45.

${ }^{16}$ M. Fujita, A. Sakai, and T. Baba, IEEE J. Sel. Top. Quantum Electron. 5, 673 (1999).

${ }^{17}$ J. Vučković, O. Painter, Y. Xu, A. Yariv, and A. Scherer, IEEE J. Sel. Top. Quantum Electron. 35, 1168 (1999). 